\title{
Determination of Macrolide Antibiotics Using Dispersive Liquid-Liquid Microextraction Followed by Surface-Assisted Laser Desorption/ Ionization Mass Spectrometry
}

\author{
Kuan-Yu Chen, Thomas C. Yang, Sarah Y. Chang
}

Department of Chemistry, National Kaohsiung Normal University, no. 62, Shenjhong Rd., Yanchao, Kaohsiung, Taiwan 824

\begin{abstract}
A novel method for the determination of macrolide antibiotics using dispersive liquid-liquid microextraction coupled to surface-assisted laser desorption/ionization mass spectrometric detection was developed. Acetone and dichloromethane were used as the disperser solvent and extraction solvent, respectively. A mixture of extraction solvent and disperser solvent were rapidly injected into a $1.0 \mathrm{~mL}$ aqueous sample to form a cloudy solution. After the extraction, macrolide antibiotics were detected using surface-assisted laser desorption/ionization mass spectrometry (SALDI/MS) with colloidal silver as the matrix. Under optimum conditions, the limits of detection (LODs) at a signal-to-noise ratio of 3 were 2, 3, 3, and $2 \mathrm{nM}$ for erythromycin (ERY), spiramycin (SPI), tilmicosin (TILM), and tylosin (TYL), respectively. This developed method was successfully applied to the determination of macrolide antibiotics in human urine samples.
\end{abstract}

Key words: Dispersive liquid-liquid microextraction, Macrolide antibiotics, SALDI/MS, Urine sample

\section{Introduction}

$\mathrm{M}$ acrolides are lipophilic molecules that contain a central lactone ring bearing 12-16 carbon atoms to which several amino and/or neutral sugars are bound. They are medium-spectrum antibiotics and are active against both gram-positive and gram-negative bacteria. Macrolides are widely used in human and veterinary medicine to prevent and treat microbial infections [1]. Different methods have been proposed for analyzing macrolides [2-4]. Among these methods, LC/MS is the most popular technique because of its sensitivity, specificity, and its ability to identify unknowns. However, LC/MS requires lengthy analyses and high cost.

The difficulties in establishing analytical methods for the analysis of macrolides in food and biological samples are mainly attributable to the complexity of the sample matrices and the low concentrations of the macrolides in the samples.

Correspondence to: Sarah Y. Chang; e-mail: ychang@nknucc.nknu.edu.tw
Therefore, an extraction/preconcentration step is generally required for the determination of macrolides in complex sample matrices. Liquid-liquid extraction (LLE) [5] and solid-phase extraction (SPE) [6] have become commonly utilized procedures to extract macrolides from urine and water samples. In addition, pressurized liquid extraction [7] and dispersive solid-phase extraction [8] have also been reported to extract macrolides from food samples. However, these sample pretreatment procedures are tedious and timeconsuming. Recently, dispersive liquid-liquid microextraction (DLLME) has become an important sample preparation technique because of its rapidity, ease of operation, and low cost [9]. Despite the wide applicability of DLLME to pesticides and polyaromatic hydrocarbons (PAHs), there are few reports on the use of DLLME of pharmaceutical compounds in the literature [10]. Additionally, DLLME has not yet been applied to the extraction of macrolides.

In surface-assisted laser desorption/ionization mass spectrometry (SALDI/MS), nanomaterials such as gold nanoparticles (NPs) [11], silver NPs [12], titanium dioxide NPs 
[13], and colloidal graphite [14] are used as SALDI matrices. These inorganic matrices provide a number of advantages over conventional organic matrices, including ease of sample preparation, lower background noise in the low-mass region, and high spot-to-spot reproducibility. Therefore, SALDI/MS is a practical tool for quantifying drugs in biological samples. However, complex sample matrices and low analyte concentrations have hampered its use in clinical drug monitoring. To overcome these limitations, we combined the DLLME technique with SALDI/MS detection and investigated the factors that influence the extraction efficiency and analyte determination. The applicability of the method to the determination of macrolides in human urine was also demonstrated. To our knowledge, this is the first report that demonstrates the use of DLLME coupled to SALDI/MS for the analysis of macrolides.

\section{Experimental}

\section{Chemicals}

Erythromycin, tilmicosin, tylosin tartrate, roxithromycin, and dichloromethane were purchased from Sigma (St. Louis, MO, USA). Spiramycin was obtained from Fluka (St. Louis, MO, USA). A colloidal suspension of silver (20 ppm) was purchased from Purest Colloids (Westhampton, NJ, USA). All chemicals were used as received without further purification. Water that was purified with a Millipore Synergy water purification system (Billerica, MA, USA) was used for all solutions.

\section{DLLME Procedure}

An aliquot $(1 \mathrm{~mL})$ of buffered solution containing the macrolides was placed in a $1.5 \mathrm{~mL}$ sample vial. A total of $200 \mu \mathrm{L}$ of acetone, the disperser solvent, containing $30 \mu \mathrm{L}$ of dichloromethane, the extraction solvent, was rapidly injected into the sample solution using a $1.0 \mathrm{~mL}$ syringe (Hamilton, Nevada, USA), and a cloudy solution was formed. The mixture was gently shaken for $5 \mathrm{~min}$. The mixture was then centrifuged at $4000 \mathrm{~g}$ for $5 \mathrm{~min}$, and the dispersed fine droplets of the extraction solvent settled at the bottom of the sample vial. Twenty microliters of the sediment phase was transferred to a separate sample vial using a $25 \mu \mathrm{L}$ HPLC syringe (Hamilton, Nevada, USA).

\section{SALDI/MS Measurements}

The extract was evaporated to dryness and re-dissolved with $2.5 \mu \mathrm{L}$ of colloidal silver solution. Then, $1 \mu \mathrm{L}$ of the mixture was deposited onto a stainless steel target and was allowed to dry at room temperature. The SALDI/MS system (Microflex; Bruker Daltonics, Bremen, Germany) has been described previously [12].

\section{Results and Discussion}

\section{Determination of Macrolides by SALDI/MS}

Previously, silver NPs and colloidal silver were found to be efficient SALDI matrices for the ionization of lipophilic molecules, such as $n$-alkanes [15], olefines [16], and estrogens [17]. We analyzed four macrolides, including erythromycin, spiramycin, tilmicosin, and tylosin to investigate the use of colloidal silver as an effective SALDI matrices. Figure 1a shows the mass spectra of the four macrolides with ion signals at $\mathrm{m} / \mathrm{z}=756.41,865.60,891.60$, and 938.56, which corresponded to the sodium adduct ions of erythromycin, spiramycin, tilmicosin, and tylosin, respectively. Although $[\mathrm{Ag}]^{+},[2 \mathrm{Ag}]^{+}$, and $[3 \mathrm{Ag}]^{+}$contributed some background ions, the results indicated that colloidal silver could be used as SALDI matrix for the detection of macrolides. In comparison, we used $\alpha$-cyano-4-hydroxycinnamic acid ( $\alpha$-CHCA), a common organic matrix for MALDI/MS, to facilitate the ionization of the macrolides. As shown in Figure 1b, macrolides were predominantly ionized as $[\mathrm{M}+\mathrm{H}]^{+}$, but signals for the sodium and potassium adducts of the macrolides were also present. The signals were observed at $\mathrm{m} / \mathrm{z} 756.41,772.42,843.58$, $865.60,869.64,891.60,916.60$, and 938.56, which corresponded to $[\mathrm{ERY}+\mathrm{Na}]^{+}$, $[\mathrm{ERY}+\mathrm{K}]^{+}$, $[\mathrm{SPI}+\mathrm{H}]^{+}$, [SPI + $\mathrm{Na}]^{+},[\text {TILM }+\mathrm{H}]^{+},[\text {TILM }+\mathrm{Na}]^{+},[\mathrm{TYL}+\mathrm{H}]^{+}$, and $[\mathrm{TYL}+$ $\mathrm{Na}]^{+}$, respectively. In addition, the peak at $m / z=738.48$ was assigned as $\left[\mathrm{ERY}+\mathrm{Na}-\mathrm{H}_{2} \mathrm{O}\right]^{+}$. Because erythromycin is sensitive to acidic solutions, the degradation product of erythromycin was detected when using $\alpha$-CHCA as the matrix [18]. Compared with $\alpha$-CHCA, the use of colloidal silver as a SALDI matrix had less complex mass spectra and fewer problems associated with inhomogeneous samples.

\section{Optimization of DLLME}

To optimize the extraction efficiency of DLLME, erythromycin was chosen as a model analyte. The extraction efficiency of erythromycin was monitored based on the

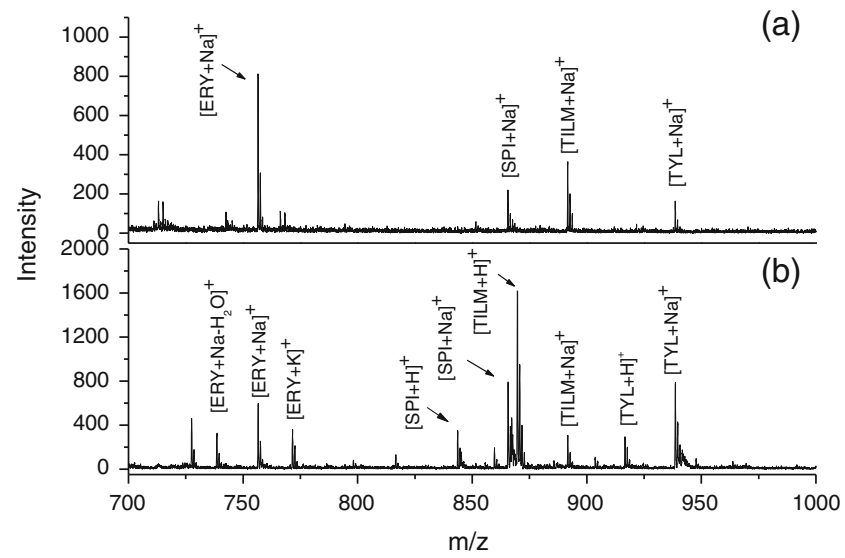

Figure 1. Mass spectra of erythromycin, spiramycin, tilmicosin, and tylosin with (a) colloidal silver, (b) $\alpha-C H C A$ matrices. The sample solutions were prepared in $5 \mathrm{mM}$ phosphate buffer, $\mathrm{pH} 10$. The concentrations of the four macrolides were $30 \mu \mathrm{M}$. A total of 50 pulsed laser shots were applied using a laser that was set to $30 \mu \mathrm{J} /$ pulse 
Table 1. Analytical Characteristics of the Four Macrolides

\begin{tabular}{lccrr}
\hline Analyte & Linear range $(\mu \mathrm{M})$ & Regression equation ${ }^{\mathrm{a}}$ & $\mathrm{r}^{\mathrm{b}}$ & $\mathrm{LOD}^{\mathrm{c}}(\mathrm{nM})$ \\
\hline Erythromycin & $0.01 \sim 3.00$ & $\mathrm{y}=16.06 \mathrm{x}+0.39$ & 0.9984 & 2 \\
Spiramycin & $0.01 \sim 2.50$ & $\mathrm{y}=14.55 \mathrm{x}+0.03$ & 0.9978 & 3 \\
Tilmicosin & $0.01 \sim 3.00$ & $\mathrm{y}=7.86 \mathrm{x}+0.03$ & 0.9977 & 122 \\
Tylosin & $0.01 \sim 3.00$ & $\mathrm{y}=7.12 \mathrm{x}+0.12$ & 0.9964 & 144 \\
\hline
\end{tabular}

${ }^{\mathrm{a}} \mathrm{y}$, peak area ratio of analyte/I.S.; $\mathrm{x}$, analyte concentration.

${ }^{b}$ Correlation coefficient.

${ }^{c}$ Limit of detection, $\mathrm{S} / \mathrm{N}=3$.

${ }^{d}$ The concentrations of erythromycin and spiramycin were $0.3 \mu \mathrm{M}$, and the concentrations of tilmicosin, and tylosin were $0.5 \mu \mathrm{M}$.

absolute ion intensities of sodium adduct ions in three samples. The selection of disperser solvent and extraction solvent are critical factors in DLLME. All combinations of dichloromethane, carbon tetrachloride, and chlorobenzene as extraction solvents with acetonitrile, ethanol, and acetone as disperser solvents were investigated. Thirty microliters of extraction solvent was dissolved in $200 \mu \mathrm{L}$ of the disperser solvent. When ethanol was used as the disperser solvent, no stable cloudy solution was formed. The combination of dichloromethane and acetone provided the highest extraction efficiency. The effect of disperser solvent volume on the signal intensity was investigated. The results showed that the signal intensities increased with increasing disperser solvent volume from 110 to $200 \mu \mathrm{L}$. At lower volumes of acetone, the cloudy solution did not form well, resulting in a decrease in the signal intensities. At higher volumes of acetone, the solubility of the erythromycin in water increased, and the signal intensities decreased. To investigate the effect of the extraction solvent volume, different volumes of dichloromethane $(30,40,50,60$, and $70 \mu \mathrm{L})$ were subjected to the same DLLME procedures. The results indicated that an increase in the extraction solvent volume resulted in a decrease in the signal intensities. This could be attributed to the formation of larger dichloromethane droplets and consequently increased sediment phase volumes. Hence, $30 \mu \mathrm{L}$ of extraction solvent volume was used for all subsequent experiments.

The $\mathrm{pH}$ of the aqueous solution significantly affects the analyte extractions in DLLME. The pKa values of the four macrolides are 7.4 to 8.9 [19]; therefore, the extractions of macrolides were examined under different $\mathrm{pH}$ conditions that ranged from 7.0 to 10.0 . The maximum extraction efficiencies all occurred at $\mathrm{pH} 10.0$ for the four macrolides. The macrolides carried 1 to 2 positive charges at $\mathrm{pH}$ 7.0. By increasing the $\mathrm{pH}$ of solution from 7.0 to 10.0 , the signal intensities of the four macrolides increased because of the decreases of positive charges on the macrolide structures. At $\mathrm{pH} \mathrm{10.0,} \mathrm{the} \mathrm{four} \mathrm{macrolides} \mathrm{were} \mathrm{neutral} \mathrm{molecules} \mathrm{and}$ were extracted efficiently with DLLME. The optimal extraction time was also investigated by plotting the signal intensities of erythromycin versus the extraction time in the range of 2-30 min while holding all other parameters constant. As expected, the observed signal intensities of erythromycin gradually increased with increasing extraction times from 2 to $5 \mathrm{~min}$, and reached a plateau after $5 \mathrm{~min}$. Thus, the extraction time was set to $5 \mathrm{~min}$.

\section{Analytical Characteristics}

Roxithromycin (ROX) was used as an internal standard (I.S.). We examined the reproducibility of the SALDI sample preparation. The signal intensities of the sodium adduct ions of erythromycin varied $10.6 \%$ over 21 sample spots (three samples) in the presence of internal standard. The peak area ratio of analyte/I. S. was used to construct calibration curve. Calibration curves for erythromycin, tilmicosin, and tylosin in aqueous solutions were over the concentration range from 0.01 to $3.00 \mu \mathrm{M}$. For spiramycin, the concentration range of the calibration curve was from 0.01 to $2.50 \mu \mathrm{M}$. The results are summarized in Table 1 . Within the concentration range studied, a good linear correlation $(\gamma>0.99)$ between the peak area ratio of analyte/I.S. and concentration was obtained for each analyte. The linear range covered more than two orders of magnitude of concentrations. The concentration LODs were calculated based on a S/N ratio of 3, which were 2, 3, 3, and $2 \mathrm{nM}$ for

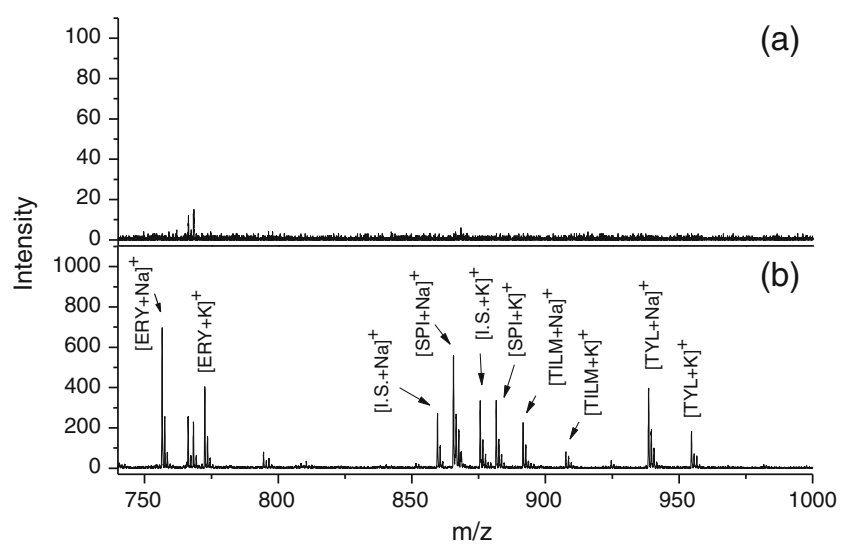

Figure 2. Mass spectra of human urine samples obtained (a) without pretreatment and (b) after extraction by DLLME. Experimental conditions: urine sample volume, $1 \mathrm{~mL}(\mathrm{pH} 10)$; organic extractant, dichloromethane $(30 \mu \mathrm{L})$; dispersive solvent, acetone (200 $\mu \mathrm{L})$; extraction time, $5 \mathrm{~min}$; spiramycin, tilmicosin, and tylosin concentration spiked, $6 \mu \mathrm{M}$; and erythromycin concentration spiked, $3 \mu \mathrm{M}$. The other conditions were the same as in Figure 1 
erythromycin, spiramycin, tilmicosin, and tylosin, respectively. Enrichment factor (EF) was estimated following the previously reported method [20]. EFs for erythromycin, spiramycin, tilmicosin, and tylosin were 122, 144, 167, and 171 , respectively.

\section{Applications}

To evaluate the applicability of the method for biological and clinical analysis, human urine spiked with erythromycin, spiramycin, tilmicosin, and tylosin was used as the test sample. The macrolide-spiked urine $(1 \mathrm{~mL})$ was treated with DLLME, following the procedure described above. Typical mass spectra obtained from the spiked urine samples are shown in Figure 2a. Without DLLME, no macrolide ions were detected. Samples from biological sources often contain high salt concentration, and the presence of these salts significantly suppresses the analyte signals in SALDI/MS. When using DLLME to extract the macrolides from the urine sample, the sodium and potassium adduct ions of the four analytes were detected in the mass spectrum (Figure 2b). The calibration curves showed good linearity over the concentration range of 0.06 to $2.50 \mu \mathrm{M}$ for spiramycin and tylosin and from 0.01 to $2.00 \mu \mathrm{M}$ for erythromycin. The linear range of tilmicosin was from $0.05-3.00 \mu \mathrm{M}$. At an $\mathrm{S} / \mathrm{N}$ ratio of 3 , the LODs for erythromycin, spiramycin, tilmicosin, and tylosin in urine were calculated to be $3,16,13$, and $17 \mathrm{nM}$, respectively. Recoveries of macrolides in urine samples were determined by standard addition method. Based on triplicate measurements, the mean recoveries of erythromycin, spiramycin, tilmicosin, and tylosin were found to be $46.3 \%, 48.2 \%, 54.7 \%$, and $55.9 \%$, respectively. In the future, the recovery may be improved by using organic solvent with higher polarity or ionic liquid. The use of DLLME effectively eliminated the analyte signal suppression that was due to the complex biological matrix. The analysis of macrolides in urine sample can be readily performed in less than $10 \mathrm{~min}$. This developed SALDI/MS method, with its speed and ease of operation, is suitable for highthroughput screening of macrolides in human urine samples.

\section{Acknowledgments}

The authors acknowledge financial supported for this work by the National Science Council (NSC 99-2113-M-017-002-MY2) of Taiwan.

\section{References}

1. Tenover, F.C.: Mechanisms of antimicrobial resistance in bacteria. Am. J. Infect. Control 34, S3-S10 (2006)

2. González dela Huebra, M.J., Veincent, U.: Analysis of macrolide antibiotics by liquid chromstography. J. Pharm. Biomed. Anal. 39, 376 398 (2005)

3. Liu, Y.-M., Shi, Y.-M., Liu, Z.-L., Tian, W.: A sensitive method for simultaneous determination of four macrolides by $\mathrm{CE}$ with electro- chemiluminescence detection and its applications in human urine and tablets. Electrophoresis 31, 364-370 (2010)

4. Wang, J., Leung, D., Lenz, S.P.: Determination of five macrolide antibiotic residues in raw milk using liquid chromatography-electrospray ionization tandem mass spectrometry. J. Agric. Food Chem. 54, 2873-2880 (2006)

5. González de la Huebra, M.J., Bordin, G., Rodríguez, A.R.: A multiresidue method for the simultaneous determination of 10 macrolide antibiotics in human urine based on gradient elution liquid chromatography coupled to coulometric detection. Anal. Chim. Acta 517, 53-63 (2004)

6. Yang, S., Carlson, K.H.: Solid-phase extraction-high-performance liquid chromatography-ion trap mass spectrometry for analysis of trace concentrations of macrolide antibiotics in natural and waste water matrices. J. Chromatogr. A 1038, 141-155 (2004)

7. Berrada, H., Borrull, F., Font, G., Marcé, R.M.: Determination of macrolide antibiotics in meat and fish using pressurized liquid extraction and liquid chromatography-mass spectrometry. J. Chromatogr. A 1208, 83-89 (2008)

8. Boscher, A., Guignard, C., Pellet, T., Hoffmann, L., Bohn, T.: Development of a multi-class method for the quantitation of veterinary drug residues in feedingstuffs by liquid chromatography-tandem mass spectrometry. J. Chromatogr. A 1217, 6394-6404 (2010)

9. Herrera-Herrera, A.V., Asensio-Ramos, M., Hernández-Borges, J., Rodríguez-Delgado, M.A.: Dispersive liquid-liquid microextraction for determination of organic analytes. Trends Anal. Chem. 29, 728$751(2010)$

10. Xiong, C., Ruan, J., Cai, Y., Tang, Y.: Extraction and determination of some psychotropic drugs in urine samples using dispersive liquid-liquid microextraction followed by high-performance liquid chromatography. J. Pharm. Biomed. Anal. 49, 572-578 (2009)

11. Su, C.-L., Tseng, W.-L.: Gold nanoparticles as assisted matrix for determining neutral small carbohydrates through laser desorption/ ionization time-of-flight mass spectrometry. Anal. Chem. 79, 16261633 (2007)

12. Wang, M.-T., Liu, M.-H., Wang, C.R.C., Chang, S.Y.: Silver-coated gold nanoparticles as concentrating probes and matrices for surfaceassisted laser desorption/ionization mass spectrometric analysis of aminoglycosides. J. Am. Soc. Mass Spectrom. 20, 1925-1932 (2009)

13. Lee, K.-H., Chiang, C.-K., Lin, Z.-H., Chang, H.-T.: Determining enediol compounds in tea using surface-assisted laser desorption/ ionization mass spectrometry with titanium dioxide nanoparticle matrices. Rapid Commun. Mass Spectrom. 21, 2023-2030 (2007)

14. Zhang, H., Cha, S., Yeung, E.S.: Colloidal graphite-assisted laser desorption/ionization $\mathrm{MS}$ and $\mathrm{MS}^{\mathrm{n}}$ of small molecules. 2. Direct profiling and MS imaging of small metabolites from fruits. Anal. Chem. 79, 6575-6584 (2007)

15. Sluszny, C., Yeung, E.S., Nikolau, B.J.: In-situ probing of the bioticabiotic boundary of plants by laser desorption/ionization time-of-flight mass spectrometry. J. Am. Soc. Mass Spectrom. 16, 107-115 (2005)

16. Sherrod, S.D., Diaz, A.J., Russell, W.K., Cremer, P.S., Russell, D.H.: Silver nanoparticles as selective ionization probes for analysis of olefins by mass spectrometry. Anal. Chem. 80, 6796-6799 (2008)

17. Chiu, T.-C., Chang, L.-C., Chiang, C.-K., Chang, H.-T.: Determination estrogen using surface-assisted laser desorption/ionization mass spectrometry with silver nanoparticles as the matrix. J. Am. Soc. Mass Spectrom. 19, 1343-1346 (2008)

18. Hirsch, R., Ternes, T., Haberer, K., Kratz, K.-L.: Occruance of antibiotics in the aquatic environment. Sci. Total Environ. 225, 109118 (1999)

19. Yudthavorasit, S., Chiaochan, C., Leepipatpiboon, N.: Simultaneous determination of multi-class antibiotics residues in water using carriermediated hollow-fiber liquid-phase microextraction coupled with ultrahigh performance liquid chromatography tandem mass spectrometry. Microchim. Acta 172, 39-49 (2011)

20. Hsu, C.-C., Whang, C.-W.: Microscale solid phase extraction of glyphosate and aminomethylphosphonic acid in water and guava fruit extract using alumina-coated iron oxide nanoparticles followed by capillary electrophoresis and electrochemiluminescence detection. $J$. Chromatogr. A 1216, 8575-8580 (2009) 